\title{
Relative Amounts of Antagonistic Splicing Factors, hnRNP A1 and ASF/SF2, Change During Neoplastic Lung Growth: Implications for Pre-mRNA Processing
}

\author{
Laura K. Zerbe, ${ }^{1 \dagger}$ Irene Pino, ${ }^{2 \dagger}$ Ruben Pio, ${ }^{2}$ Pippa F. Cosper, ${ }^{1}$ Lori D. Dwyer-Nield, ${ }^{1}$ Amy M. Meyer, ${ }^{3}$ \\ J. David Port, ${ }^{3}$ Luis M. Montuenga, ${ }^{2}$ and Alvin M. Malkinson ${ }^{1 *}$ \\ ${ }^{1}$ Department of Pharmaceutical Sciences, University of Colorado Health Sciences Center, Denver, Colorado \\ ${ }^{2}$ Departments of Histology, Pathology, Biochemistry and Center of Applied Medical Research, \\ University of Navarra, Pamplona, Spain \\ ${ }^{3}$ Department of Pharmacology, University of Colorado Health Sciences Center, Denver, Colorado
}

Pre-mRNA processing is an important mechanism for globally modifying cellular protein composition during tumorigenesis. To understand this process during lung cancer, expression of two key pre-mRNA alternative splicing factors was compared in a mouse model of early lung carcinogenesis and during regenerative growth following reversible lung injury. Heterogeneous nuclear ribonucleoprotein (hnRNP) A1 and alternative splicing factor/ splicing factor 2 (ASF/SF2) act antagonistically to modulate splice site selection. Both hnRNP A1 and ASF/SF2 contents rose in adenomas and during injury-induced hyperplasia compared to control lungs, as measured by immunoblotting. While both proteins increased similarly during compensatory hyperplasia, hnRNP A1 increased to a much greater extent than ASF/SF2 in tumors, resulting in a 6-fold increase of the hnRNP A1 to ASF/SF2 ratio. Immunohistochemical analysis showed that hnRNP A1 localized exclusively within tumor nuclei, while ASF/SF2 appeared in cytoplasm and/or nuclei, depending on the growth pattern of the tumor cells. We also demonstrated cancer-associated changes in the pre-mRNA alternative splicing of CD44, a membrane glycoprotein involved in cell-cell and cell-extracellular matrix interactions. hnRNP A1 and ASF/SF2 expression is thus differentially altered in neoplastic lung cells by mechanisms that do not strictly arise from increased cell division. These changes are influenced by tumor histology and may be associated with production of variant CD44 mRNA isoforms. (๑) 2004 Wiley-Liss, Inc.

Key words: 3-methylcholanthrene; alternative splicing; butylated hydroxytoluene; CD44; lung cancer

\section{INTRODUCTION}

Lung cancer, the primary cause of cancer deaths in the US and Europe [1], is seldom diagnosed prior to metastatic spread. It is, therefore, critical to understand the mechanisms that guide early stages of tumor development in order to identify biomarkers and develop improved molecular-targeted chemopreventive and chemotherapeutic strategies. There is growing evidence that modulation of gene expression at the pre-mRNA level is an important regulatory mechanism in tumorigenesis [2]. Pre-mRNA processing proteins mediate many aspects of preparing a final mRNA transcript for polysomal translation, including alternative splicing, stability, and transport of pre-mRNA [3,4], and thus can influence the translation into proteins of numerous mRNAs, including those that facilitate carcinogenesis. Sequencing of the human genome has emphasized the importance of pre-mRNA processing, particularly alternative splicing, in normal as well as pathological tissues. The discovery of far fewer genes than anticipated based on mammalian proteome complexity indicates that alternative splicing and posttranslational modifications are critical for protein diversity [5]. In fact, it is estimated that $40-60 \%$ of genes are alternatively spliced into two or more transcripts [6]. Computational analysis of mRNAs derived from tumor or normal samples identified 845 alternatively spliced isoforms significantly associated with human cancer [7].

At least 20 hnRNP proteins in human cells form a diverse set of large, complexes with heterogeneous

Abbreviations: AC, adenocarcinoma; ASF/SF2, alternative splicing factor/splicing factor 2; BHT, butylated hydroxytoluene; hnRNP, heterogeneous nuclear ribonucleoprotein; IHC, immunohistochemistry; MCA, 3-methylcholanthrene; SR, serine/arginine.

"Laura K. Zerbe and Irene Pino contributed equally to this study.

*Correspondence to: Department of Pharmaceutical Sciences, University of Colorado Health Sciences Center, Box C238, 4200 East Ninth Ave., Denver, CO 80262.

Received 8 April 2004; Revised 1 July 2004; Accepted 20 July 2004

DOI 10.1002/mc.20053 
nuclear RNA during pre-mRNA processing [3]. The pre-mRNA processing protein, heterogeneous nuclear ribonucleoprotein (hnRNP) A2/B1, has been proposed as a promising biomarker for lung cancer. In prospective studies, hnRNP A2/B1 expression in epithelial cells exfoliated into human sputum predicted lung cancer in approximately $70 \%$ of the subjects [8]. We demonstrated previously by immunohistochemistry (IHC) that several members of this family (hnRNP A1, A2, B1, C1, C2, and K) are increased in tumors compared to normal tissue from lung cancer patient biopsies [9]. We have also shown that several pre-mRNA processing proteins, hnRNP A2/B1 [10], hnRNP D, and Hu antigen R (HuR) [11], increase in mouse lung tumors and related neoplastic cell lines, which are preclinical models of pulmonary adenocarcinoma [12].

Serine/arginine (SR)-rich proteins, another important class of pre-mRNA processing proteins involved in constitutive and alternative splicing, interact with the hnRNP proteins. One member of this family, alternative splicing factor/splicing factor 2 (ASF/ SF2), acts antagonistically to hnRNP A1 to regulate alternative splicing; increased concentrations of ASF/SF2 select proximal $5^{\prime}$ sites, while high hnRNP A1 levels promote distal $5^{\prime}$ site selection $[13,14]$. Several SR proteins, including ASF/SF2, increase during mouse mammary gland tumorigenesis [15].

Because of the increased expression patterns of hnRNP proteins in human lung cancer and the known importance of interactions between hnRNP A1 and ASF/SF2 in regulating alternative splicing, we evaluated expression of these two proteins in mouse models of lung AC and lung regenerative repair. Our results indicate that both hnRNP A1 and ASF/SF2 increase in benign tumors and in nonneoplastic proliferating lung compared to normal lung tissue. In tumors, however, hnRNP A1 increased substantially more than ASF/SF2, while both proteins increased similarly during regenerative hyperplasia. To demonstrate that alternative splicing indeed occurs in this lung cancer model, we evaluated the mRNA isoform pattern of CD44, a gene whose alternative splicing is associated with many cancers [16-18]. Interestingly, the increased ratio of hnRNP A1 relative to ASF/SF2 in tumors corresponded to the presence of metastasis-associated CD44 splice variants relative to control lung, while no changes in CD44 splicing were observed in nonneoplastic proliferating cells.

\section{MATERIALS AND METHODS}

Mice and Treatments

Male BALB/cByJ mice were obtained from Jackson Laboratories (Bar Harbor, ME) at 5-7 weeks of age, and allowed to acclimate for 10-14 days prior to their use in experiments. The mice were given access to Teklad-8640 standard laboratory chow (Harlan
Teklad, Madison, WI) and water ad libitum, and maintained on a hardwood bedding under a $12 \mathrm{~h}$ light/12 $\mathrm{h}$ dark cycle. All injections were given prior to 10 AM because of circadian effects on BHT pneumotoxicity (Malkinson, A.M., unpublished results). Mice were sacrificed by lethal injection of $100 \mu \mathrm{l} 90 \mathrm{mg} / \mathrm{ml}$ sodium pentobarbital containing 1000 U/ml heparin (Sigma, St. Louis, MO).

\section{MCA/BHT 2-Stage Lung Carcinogenesis in BALB Mice}

Mice were injected IP once with $15 \mathrm{mg} / \mathrm{kg}$ body wt MCA (Sigma) dissolved in Mazola ${ }^{\mathrm{TM}}$ corn oil (ACH Food Companies, Memphis, TN), followed by 6 weekly injections of BHT (Sigma; first injection, $150 \mathrm{mg} / \mathrm{kg}$ body $\mathrm{wt}$; the second through sixth injections, $200 \mathrm{mg} / \mathrm{kg}$ body $\mathrm{wt}$ ) dissolved in corn oil, as described [19]. Mice were sacrificed 26 wks after MCA injection.

\section{BHT-Induced Acute Lung Injury in BALB Mice}

Mice were injected IP once with $200 \mathrm{mg} / \mathrm{kg}$ body wt BHT as described [20], and sacrificed $1 \mathrm{~h}$ to 6 days after BHT administration.

\section{Preparation of Extracts for Immunoblottting}

Dissected lungs from untreated or BHT-treated mice, normal-appearing uninvolved tissue in tumorbearing lungs, and tumors were Dounce-homogenized in $100-500 \mu$ l solubilization buffer $(20 \mathrm{mM}$ HEPES, $\mathrm{pH}=7.5,2 \mathrm{mM}$ EDTA, 2 mM EGTA, $1 \mathrm{mM}$ DTT, $10 \%$ glycerol, $5 \mu \mathrm{g} / \mathrm{ml}$ aprotinin, and $10 \mu \mathrm{M}$ leupeptin), and centrifuged at $16000 \times \mathrm{g}$ for $25 \mathrm{~min}$ at $4{ }^{\circ} \mathrm{C}$. After the supernatant (soluble fraction) was removed, the pellet (particulate fraction) was resuspended in solubilization buffer containing $0.1 \%$ NP40, sonicated and an aliquot $(10-20 \mu \mathrm{l})$ was removed to determine protein concentration (based on the method of Lowry et al. [21]). FourX gelloading buffer was added to the remaining sample at a 4-fold dilution (final concentrations: $10 \%$ glycerol, $1 \%$ SDS, $50 \mathrm{mM}$ Tris, $\mathrm{pH}=6.8,4 \% \beta$-mercaptoethanol, trace amount of bromophenol blue) and incubated at $95^{\circ} \mathrm{C}$ for $5 \mathrm{~min}$. All samples were stored at $-70^{\circ} \mathrm{C}$ until immunoblot analysis.

\section{Immunoblotting}

Proteins $(25-100 \mu \mathrm{g})$ were resolved on a $15 \%$ SDSpolyacrylamide gel followed by transfer to an Immobilon-P membrane (Millipore Corp., Billerica, MA) and blocked in 3\% nonfat dried milk dissolved in TNS $(15 \mathrm{mM}$ Tris, $\mathrm{pH}=7.4,150 \mathrm{mM} \mathrm{NaCl})$ for $30 \mathrm{~min}$. The membranes was then incubated in primary anti-hnRNP A1 monoclonal antibodies (4B10 or 9H10, kindly supplied by Dr. G. Dreyfuss, HHMI, University of Pennsylvania) diluted 1:1000 in TNS or anti-ASF/SF2 goat polyclonal antibody P-15, sc-10254 (Santa Cruz Biotechnology, Santa Cruz, CA) diluted 1:100 in TNS for $1 \mathrm{~h}$ and washed in TNS 
with 3\% milk and 0.1\% Tween 20. For hnRNP A1, a secondary goat, antimouse antibody (Bio-Rad, Hercules, CA) was diluted 1:5000 in TNS, while for ASF/SF2, a rabbit antigoat antibody (A-5420, Sigma) was diluted 1:25000 in TNS and incubated for $1 \mathrm{~h}$ followed by washing in TNS with $3 \%$ milk and $0.1 \%$ Tween 20. Protein bands were visualized by chemiluminiscence after exposure to CL-Xposure film (Pierce, Rockford, IL) with Immun-Star ${ }^{\mathrm{TM}}$ AP substrate (Bio-Rad) for hnRNP A1 and Western Lighting (PE Applied Biosystems, Foster, CA) for ASF/SF2. Immunoreactive proteins on Western blots were quantified by UN-SCAN-IT ${ }^{\mathrm{TM}}$ (Silk Scientific, Orem, UT). Ponceau-staining $(0.1 \%$ ponceau in $5 \%$ acetic acid) of proteins transferred to the membrane confirmed equal protein loading.

\section{Lung Fixation}

Immediately after the mice were sacrificed, the lungs were gently inflated through a canulated trachea with formalin for $1 \mathrm{~h}$. Lungs were then removed from the chest cavity and individual lobes dissected and submerged in formalin overnight. Fixed lungs were transferred to $95 \%$ ethanol and embedded in paraffin.

\section{Immunohistochemistry}

Tissue sections $(3 \mu \mathrm{m})$ were deparaffinized and endogenous peroxidase activity blocked by treatment with $3 \%$ hydrogen peroxide. For hnRNP A1, after pretreatment with the Histomouse-SP ${ }^{\mathrm{TM}}$ Blocker Kit (Zymed Laboratories, Inc., San Francisco, CA), 4B10 primary antibody was used at a 1:1000 dilution overnight at $4^{\circ} \mathrm{C}$. Slides were rinsed and incubated with the ENVISION ${ }^{\mathrm{TM}}$ reagent for mouse monoclonal antibodies (Dakocytomation, Glostrup, Denmark) for $30 \mathrm{~min}$ at room temperature. For ASF/ SF2 immunostaining, slides were immersed in $10 \mathrm{mM}$ citrate buffer, $\mathrm{pH} \mathrm{6}$, and microwaved at two 15-min cycles to retrieve antigen before blocking with rabbit normal serum (1:20; Dakocytomation). The sections were incubated with a primary goat polyclonal antibody P-10254 (Santa Cruz Biotechnology) diluted $1: 100$ at $4^{\circ} \mathrm{C}$ overnight. Addition of an antigoat rabbit secondary biotinylated antibody (1:300; Dakocytomation) was followed by ABC complex incubation (1:100; Dakocytomation). Immunostaining was developed with diaminobenzidine (Dakocytomation) and counter-stained with hematoxylin.

\section{RT-PCR}

Freshly prepared dissected tumor, uninvolved lung from tumor-bearing mice or control lung tissue was submerged in $0.5-1 \mathrm{~mL}$ ice-cold RNAlater (Ambion, Austin, TX) and stored at $-20^{\circ}$ until future analysis. RNA was subsequently isolated with either TRIzol reagent (Invitrogen, Carlsbad, CA) or RNeasy mini kit (Qiagen, Valencia, CA). RNA was reverse- transcribed to DNA with the SuperScript ${ }^{\mathrm{TM}}$ firststrand synthesis system for RT-PCR (Invitrogen). CD44 isoforms were amplified with Platinum ${ }^{\mathbb{R}}$ Taq DNA polymerase (Invitrogen) and primers $5^{\prime}$ ACCCCAGAAGGCTACATTTTGC-3' (forward) and 5'-CTCATAGGACCAGAAGTTGTGG-3' (reverse) as previously described [15] at 35 rounds of PCR consisting of $45 \mathrm{~s} 94^{\circ} \mathrm{C}$, $45 \mathrm{~s} 60^{\circ} \mathrm{C}$, and $2 \min 72^{\circ} \mathrm{C}$. PCR products were separated on a $2 \%$ agarose gel, visualized with UV light and quantified by UNSCAN-IT $^{\mathrm{TM}}$ (Silk Scientific) software.

\section{Statistical Analysis}

Data were represented in graphs as mean \pm SEM and analyzed by Student's unpaired $t$-test with Prism 3.0 software (GraphPad, San Diego, CA) In all analyses, significance was accepted at $P<0.05$.

\section{RESULTS}

hnRNP A1 and ASF/SF2 Protein Expression

During 2-Stage Carcinogenesis

2-stage carcinogenesis consists of initiation by the tobacco carcinogen, MCA, followed by promotion of the growth of initiated cells by BHT. BHT undergoes extensive pulmonary metabolism in mice to generate highly reactive oxidative species that cause a reversible pneumotoxicity consisting of peripheral epithelial cell damage, regenerative growth, and inflammation; these properties promote tumor growth in some mouse strains including BALB when BHT is administered after a carcinogen $[19,20,22-$ 25]. This experimental model affords the opportunity to compare hnRNP A1 and ASF/SF2 expression during early neoplasia after 2-stage carcinogenesis $(\mathrm{MCA}+\mathrm{BHT})$ with the nonneoplastic proliferation that follows BHT treatment alone.

hnRNP A1 and ASF/SF2 protein contents were measured by immunoblot analysis of dissected tumors and uninvolved lungs (i.e., normal-appearing tissue adjacent to tumors), isolated from mice treated with MCA + BHT or whole lungs from agematched control mice (Fig. 1). hnRNP A1 levels were elevated substantially in tumor versus control particulate samples $(20300 \pm 2260$ and $700 \pm 230$ densitometric values, respectively) resulting in a 30 -fold increase. ASF/SF2 levels also increased in tumor versus control samples $(2100 \pm 146$ and $400 \pm 49$ densitometric values, respectively), but to a lesser extent than hnRNP A1. This differential increase in hnRNP A1 compared to ASF/SF2 resulted in nearly a 6-fold elevation of the hnRNP A1 to ASF/SF2 ratio. Soluble fractions were also analyzed, but negligible alterations in hnRNP A1 or ASF/SF2 contents were observed in tumor relative to control lung tissue (0.9fold to 1.4-fold increases, data not shown). Both hnRNP A1 and ASF/SF2 also rose in uninvolved compared to control tissue (3-fold and 2-fold, respectively, Fig. 1). 
A

HnRNPA1

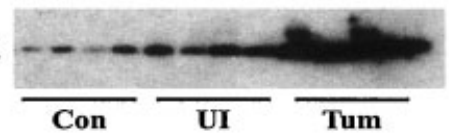

ASF/SF2

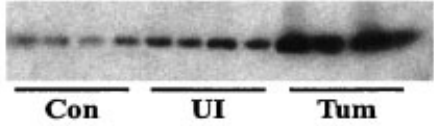

Figure 1. Expression of hnRNP A1 and ASF/SF2 protein in mouse lung tumorigenesis. Immunoblotting of hnRNP A1 (A) and ASF/SF2 (C) was conducted on particulate fractions of age-matched control lungs (Con), uninvolved lung ( $\mathrm{Ul}$; normal-appearing tissue in tumorbearing lungs) and tumors (Tum) isolated from BALB mice. Mice were treated with $15 \mathrm{mg} / \mathrm{kg}$ body wt MCA, followed by 6 weekly injections of BHT (first injection, $150 \mathrm{mg} / \mathrm{kg}$ body wt; injections 2$6=200 \mathrm{mg} / \mathrm{kg}$ body $\mathrm{wt}$ ) or 7 corn oil injections and sacrificed after

hnRNP A1 and ASF/SF2 Cellular and Intracellular Localization During 2-Stage Carcinogenesis

To determine the cellular localization of hnRNP A1 and ASF/SF2, we performed IHC on lung tissue sections obtained from BALB mice treated with $\mathrm{MCA}+\mathrm{BHT}$ (Fig. 2). hnRNP A1 was more highly expressed in tumor cells than in adjacent uninvolved alveolar and bronchiolar cells (Fig. 2A), while ASF/ SF2 levels were moderately higher in tumors compared to surrounding pulmonary tissue (Fig. 2B), similar to immunoblotting experiments (Fig. 1). Mouse lung adenomas grow in either solid or papillary patterns; papillary tumors are more aggressive with a greater tendency to progress to malignancy [26]. In both solid and papillary tumors, hnRNP A1 localized to tumor nuclei (Fig. 2C and E). In contrast, ASF/SF2 localization varied between the two pathologies; the solid phenotype contained

Figure 2. Expression and localization of hnRNP A1 and ASF/SF2 in mouse lung tumorigenesis and in nonneoplastic proliferating lung cells. Immunohistochemistry (IHC) of hnRNP A1 in solid tumors (A, C) papillary tumor (E), control lung (G), and BHT-treated lung (I). IHC of ASF/SF2 in solid tumors (B, D) and papillary tumors (F), control lung $(\mathrm{H})$, and BHT-treated lung $(J)$. Solid and papillary tumors were derived $(\mathrm{H})$, and BHT-treated lung $(\mathrm{J})$. Solid and papillary tumors were derived
from BALB mice treated with $15 \mathrm{mg} / \mathrm{kg}$ body wt MCA, followed by 6 weekly injections of BHT (first injection, $150 \mathrm{mg} / \mathrm{kg}$ body $\mathrm{wt}_{\text {; }}$
B

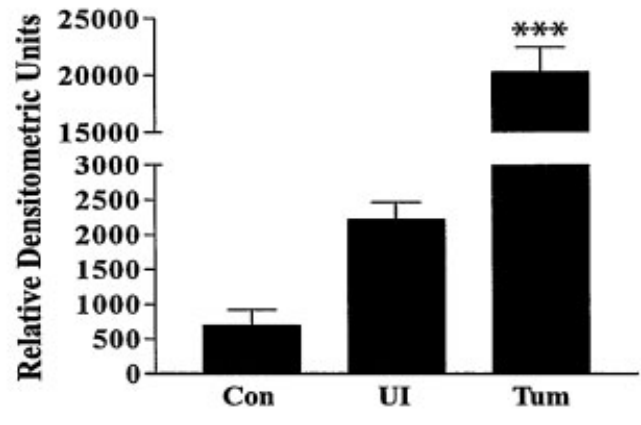

D

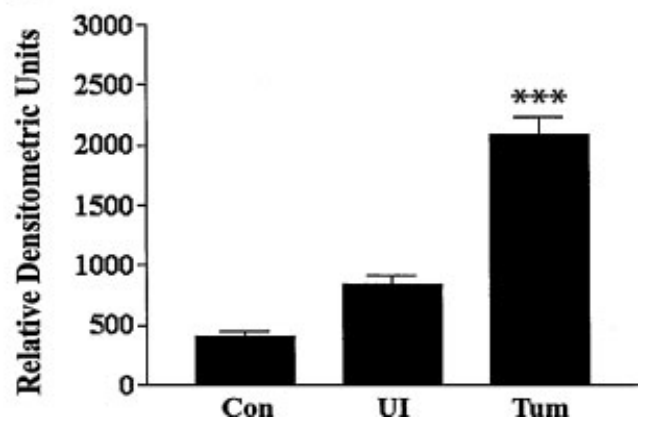

$26 \mathrm{wk}$. The bands correspond to the expected 34 and $30 \mathrm{kDa}$ molecular weights of hnRNP A1 and ASF/SF2, respectively. hnRNP A1 (B) and ASF/SF2 (D) immunoreactive protein levels were quantified by UN-SCAN-IT software. Data represent mean \pm SEM from four mice per group. For both hnRNP A1 and ASF/SF2, $* * * P \leq 0.0001$ tumor vs. control samples. Ponceau-staining of proteins transferred to the membrane confirmed equal protein loading.

both nuclear and cytoplasmic ASF/SF2 (Fig. 2D), whereas papillary tumors exhibited only cytoplasmic localization (Fig. 2F).

hnRNP A1 and ASF/SF2 Expression During Nonneoplastic Pulmonary Growth Induced by BHT

The contents of hnRNP A1 and ASF/SF2 in tumor cells may be upregulated consonant with the increased RNA metabolism found in rapidly dividing cells. To examine this, we measured hnRNP A1 and ASF/SF2 levels during nonneoplastic proliferation. BHT damage to alveolar type I cells is repaired by a regenerative growth response of Clara cells and type 2 cells; the latter cell type then differentiates into type 1 cells to repair the alveolar epithelium [25]. BALB mice were given a single dose of BHT in the absence of MCA, and lung extracts assessed by immunoblotting 6 days later in particulate fractions (Fig. 3). The contents of hnRNP A1 and ASF/SF2 both

injections $2-6=200 \mathrm{mg} / \mathrm{kg}$ body $\mathrm{wt}$ ) and sacrificed $26 \mathrm{wk}$ after MCA administration. BHT-treated and control lung samples were obtained from BALB mice injected with $200 \mathrm{mg} / \mathrm{kg}$ body wt BHT or corn oil, respectively, and sacrificed after 6 days. hnRNP A1 or ASF/ SF2 positive cells are brown. Hematoxylin counterstain. Original magnification for $A, B, C, D, E$, and $F, 1250 \times$ and for $G, H, I$, and $J$, $250 \times$ 

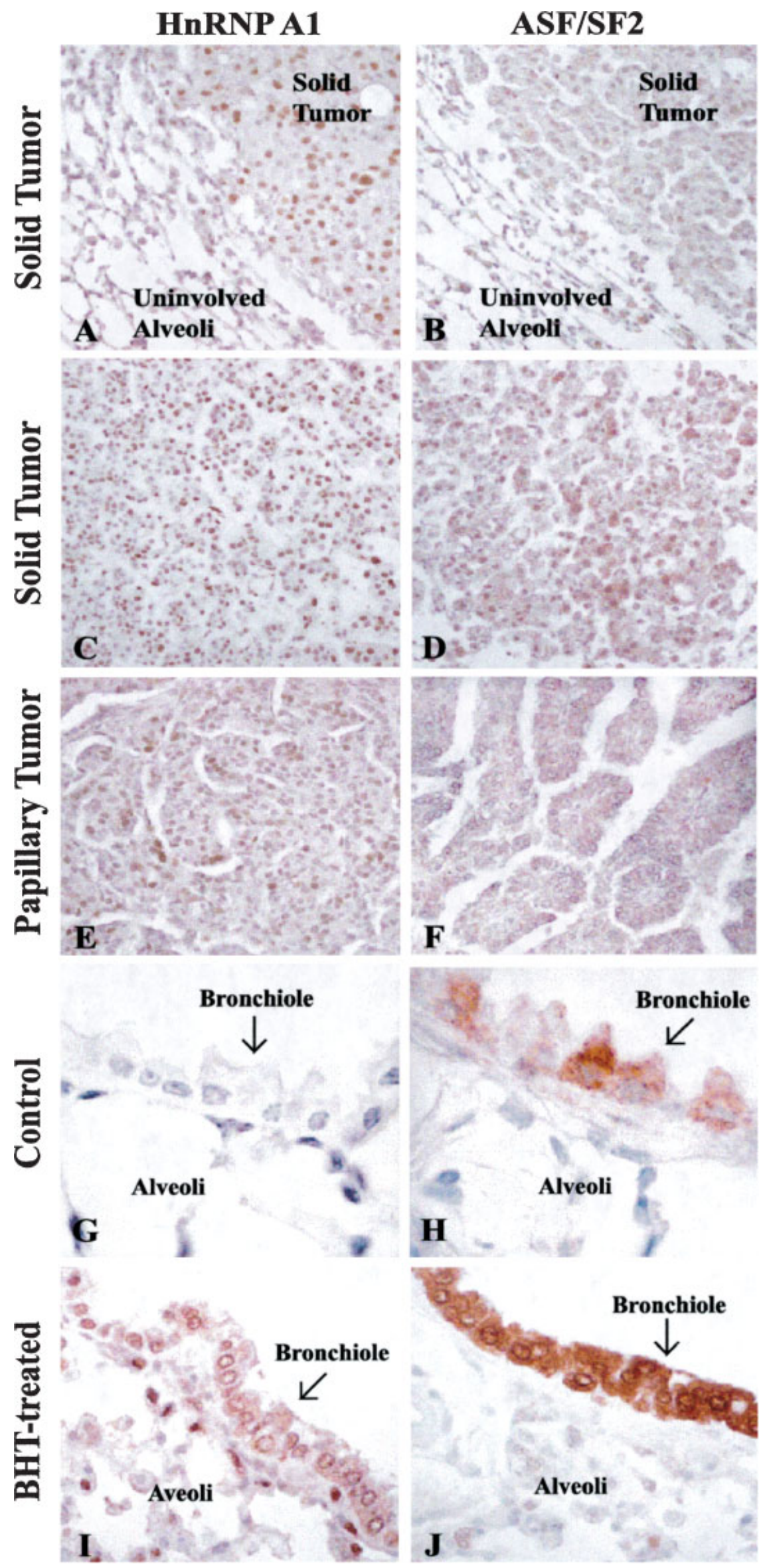

Figure 2. 
A

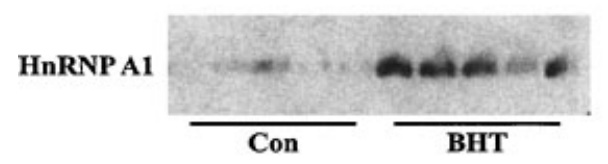

C

ASF/SF2

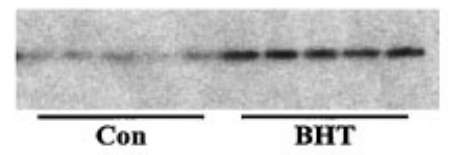

Figure 3. Expression of hnRNP A1 and ASF/SF2 protein in nonneoplastic proliferating lung cells. Immunoblotting of hnRNP A1 (A) and ASF/SF2 (C) was conducted on particulate fractions of lung tissue isolated from BALB mice treated with corn oil control (Con) or $200 \mathrm{mg} / \mathrm{kg}$ body wt BHT (BHT). Mice were sacrificed 6 days after BHT administration. The bands correspond to the expected 34 and $30 \mathrm{kDa}$ molecular weights of hnRNP A1 and ASF/SF2,

rose in BHT-treated lungs relative to control lungs to a similar extent (5.7-fold and 3.2-fold, respectively). While significant, these increases were smaller than that for hnRNP A1 in tumors relative to control lung (Fig. 1). Similar to neoplastic tissue, hnRNP A1 or ASF/SF2 expression in soluble fractions of BHTtreated versus control lung did not change (data not shown). Because hnRNP A1 and ASF/SF2 each increased to similar extents during repair of BHTinduced lung injury, the differential increases of hnRNP A1 and ASF/SF2 levels in neoplastic lung tissue probably does not simply result from increased cell division.

hnRNP A1 and ASF/SF2 Localization During Hyperplasia Induced by BHT

To determine how the cellular localization of hnRNP A1 and ASF/SF2 in nonneoplastic proliferating cells compared with their expression in tumors, IHC was performed on lungs isolated from BHTtreated BALB mice. We assayed hnRNP A1 and ASF/ SF2 expression between $1 \mathrm{~h}$ and 6 days after BHT exposure. In control mice, hnRNP A1 was not detectable by IHC in alveolar type 2 or bronchiolar Clara cells; ASF/SF2 was not detected in type 2 cells

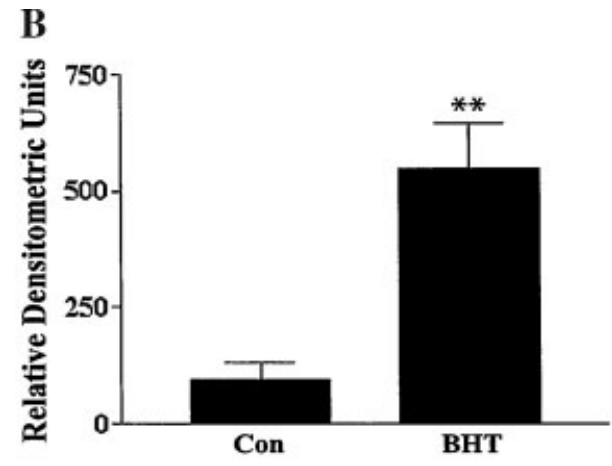

D

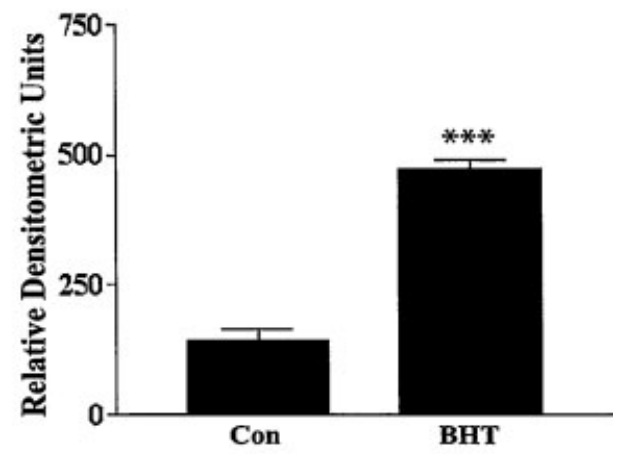

respectively. hnRNP A1 (B) and ASF/SF2 (D) immunoreactive protein levels were quantified by UN-SCAN-IT software. Data represent mean \pm SEM from five mice per group. For hnRNP A $1 * * P=0.0027$ and ASF/SF2 $* * * P<0.0001$ BHT-treated vs. control samples. Ponceau staining of proteins transferred to the membrane confirmed equal protein loading.

but was expressed in Clara cells (Fig. 2G and H). Moderate increases in alveolar hnRNP A1 and ASF/SF2 levels occurred early after BHT-treatment; cytoplasmic ASF/SF2 was detected in alveoli within $1 \mathrm{~h}$ and nuclear hnRNP A1 in alveoli by $24 \mathrm{~h}$ (data not shown). A few days after BHT-treatment, corresponding to the time of maximal cellular proliferation and repair $[20,23,25]$, high nuclear and cytosolic hnRNP A1 were detected in alveolar and bronchiolar cells (Fig. 2I). ASF/SF2 content also reached peak cytosolic and nuclear levels in alveolar and bronchiolar cells by 6 days, but bronchiolar staining was more intense (Fig. 2J). In general, the intercellular localization of ASF/SF2 was similar in nonneoplastic proliferating lung tissue and tumors, displaying both nuclear and cytosolic expression. In contrast, hnRNP A1 localization differed between the two pathologies; expression in repairing lungs was both cytoplasmic and nuclear while tumor expression was primarily nuclear.

Alternative Splicing of CD44 Pre-mRNA in Lung

Tumors and BHT-Treated Lung-A Candidate

Substrate for hnRNP A1 and ASF/SF2

The relative ratio of hnRNP A1 relative to ASF/SF2 alters the alternative splicing of several pre-mRNAs 
in vitro $[13,14]$. Given the differential increases of hnRNP A1 and ASF/SF2 in tumors relative to control tissue reported here, it seemed probable that splicing of many pre-mRNAs would be affected, including those involved in tumorigenesis. To begin to test this hypothesis, the mRNA expression pattern of $C D 44$, a gene for which alternative splicing has been implicated in many types of cancer including lung cancer [16,17], was compared in MCA-BHT lung carcinogenesis, BHT-induced nonneoplastic proliferating lung tissue, and control lung. CD44 is a cell surface molecule that regulates cell-cell and cellextracellular matrix interactions during inflammation and cancer, and is composed of 20 exons [18]. Particular variants are associated with metastasis; the CD44standard (CD44s) isoform results from splicing S1-S5 and S6-S10 exons, while metastasis-associated
CD44variant $(\mathrm{CD} 44 \mathrm{v})$ isoforms include 10 variant exons in various combinations (Fig. 4A). CD44 isoforms were measured by RT-PCR with primers that bound S5 and S6 CD44s exons and flanked the 10 CD44 variant exons V1-V10 (Fig. 4A).

In MCA + BHT studies, the mRNA expression pattern of CD44 switched from predominantly the single 221 nucleotide long CD44s exon in control and uninvolved tissues to a series of larger CD44 splice variants in tumors (Fig. 4B), similar to that observed in other tumor types $[15,16,18,27]$. In fact, $58 \%$ of the total CD44 isoforms were variant in the lung tumor extracts compared to only $14 \%$ in control tissue (Fig. 4D). The five major CD44 variant isoforms in tumors displayed a ladder pattern indicating sizes ranging from approximately 492 to 1230 nucleotides long (based on DNA ladder standards) that

A CD44 gene structure
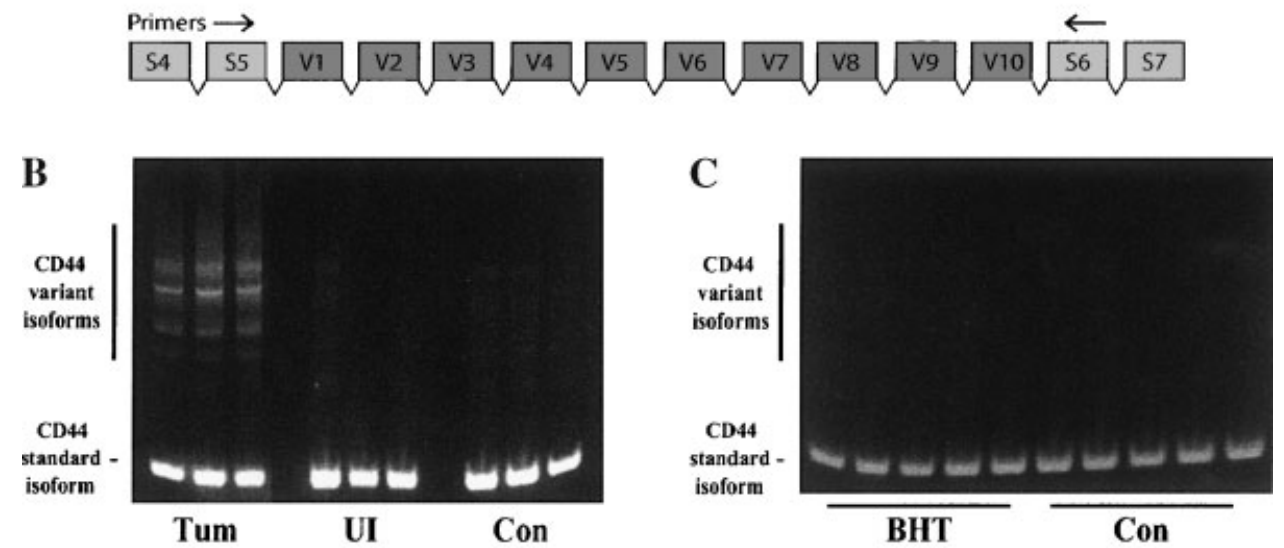

D

E

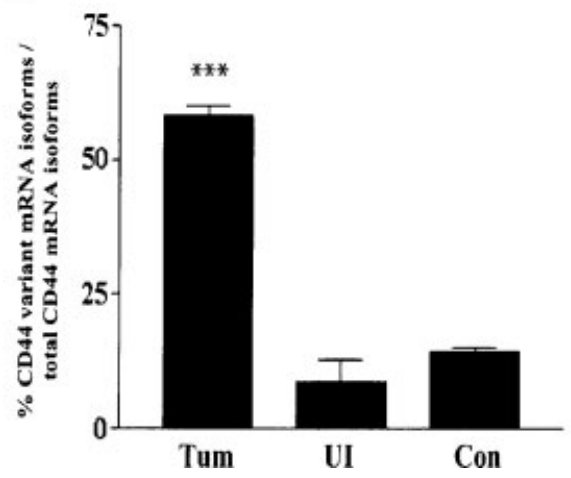

Figure 4. Expression pattern of CD44 mRNA isoforms in mouse lung pathology. The $C D 44$ gene structure showing the 10 variant exons (V1-V10) flanked by standard exons S5 and S6 (A). RT-PCR analysis of CD44 mRNA isoforms during 2-stage carcinogenesis (B) and reparative hyperplasia (C) in BALB mice with primers specific for standard exons 55 and 56 . RNA was isolated from dissected tumors (Tum), uninvolved lung (Ul; normal-appearing tissue in tumorbearing lungs), and age-matched control lungs (Con) in tumorigenesis studies (B) and from BHT-treated mice (BHT) and age-matched control lungs (Con) in reparative hyperplasia studies (C). In

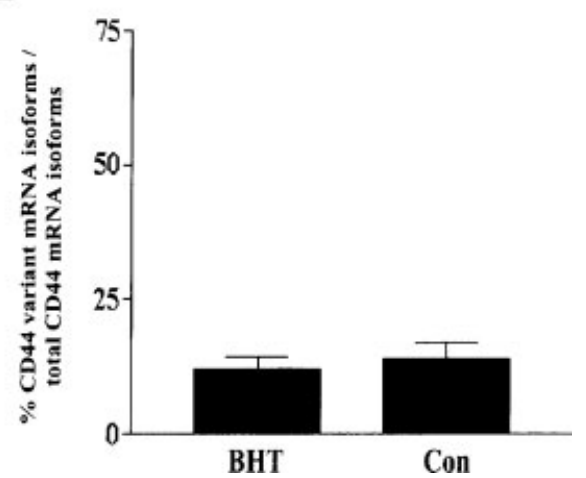

tumorigenesis studies mice were treated with $15 \mathrm{mg} / \mathrm{kg}$ body wt MCA, followed by 6 weekly injections of BHT (first injection, $150 \mathrm{mg} /$ $\mathrm{kg}$ body $\mathrm{wt}$; injections $2-6=200 \mathrm{mg} / \mathrm{kg}$ body $\mathrm{wt}$ ) or 7 corn oil injections and sacrificed after $26 \mathrm{wk}$ and in reparative hyperplasia studies mice were treated with $200 \mathrm{mg} / \mathrm{kg}$ BHT or corn oil and sacrificed after 6 days. CD44 mRNA isoforms were quantified by UNSCAN-IT software. Data represent CD44variant isoforms/CD44total isoforms $\times 100 \%$ and are the mean \pm SEM from 3 to 5 mice per group (D, E). For tumor versus control $* * * P \leq 0.0001$ (D). 
differed in size by approximately 115 nucleotides, which is comparable to previous reports $[15,27]$. In contrast, nonneoplastic proliferating lung in BHTtreated mice did not exhibit a pattern of CD44 variant isoforms; rather, the CD44s isoform predominated, similar to control lung tissue (Fig. 4C and E). These data showed that differential increases of hnRNP A1 and ASF/SF2 in mouse lung tumors correspond to an altered CD44 mRNA splice pattern, although a causal relationship is not proved.

\section{DISCUSSION}

Expression of antagonistic splicing factors, hnRNP A1 and ASF/SF2, is substantially altered in early mouse lung neoplasia. The contents of both factors rose in tumors compared to control lung, but hnRNP A1 increased more than ASF/SF2, resulting in a 6-fold increase of the hnRNP A1 to ASF/SF2 ratio. Other groups have shown in vitro that the relative levels of hnRNP A1 compared to ASF/SF2 affects $5^{\prime}$ splice site selection $[13,14]$, these differential changes in vivo probably modulate alternative splicing of many genes, including those involved in carcinogenesis. The increase in hnRNP A1 and ASF/SF2 is not exclusively due to the high proliferative activity of tumor tissues. Both factors rose in regenerating mouse lung tissue, but did not exhibit an altered hnRNP A1 to ASF/SF2 ratio. This is in agreement with our previous results showing that mRNA expression of several hnRNPs including hnRNP A1 is not correlated strictly with proliferative index in a panel of cultured human lung cell lines [9].

The aberrant expression of hnRNP A1 and ASF/SF2 occurred in early-stage tumors of mouse lung tumorigenesis (26 weeks after MCA injection) that are typically small with uniform-looking cells. Adenocarcinomas with increased disorganization of tumor cells and invasion into the stroma are not usually observed until 35 weeks after MCA treatment. This early appearance of splice factors provides evidence that they may regulate initial stages of neoplastic transformation. In fact, both ASF/SF2 and hnRNP A1 are also significantly elevated in uninvolved tissue surrounding tumors relative to control lung, suggesting that they play a role in the transition of normal cells to neoplastic. If this is the case, both factors may prove to be promising biomarkers for lung cancer, similar to hnRNP A2/B1 [8].

hnRNP A1 localized to the nucleus of tumor cells while it exhibited both nuclear and cytoplasmic localization in nonneoplastic proliferating lung (Fig. 2). ASF/SF2 was observed in both nuclear and cytoplasmic compartments in tumors as well as regenerative lung. There may be trafficking mechanisms that localize hnRNP A1 to the nucleus during tumorigenesis. Indeed, other investigators have shown that the phosphorylation status of ASF/SF2 affects its intracellular trafficking between the nucleus and cytoplasm and determines in part its nuclear content relative to hnRNP A1 $[28,29]$.

Because pre-mRNA processing proteins elicit global changes in mRNA expression, upregulation of this class of proteins in precancerous lesions can dramatically affect the neoplastic phenotype. Diversity in the proteome resulting from changes in alternative splicing would enhance the possibilities for selecting variant cells capable of overcoming normal homeostatic controls, and encourage neoplastic growth. Protein isoforms spliced from the same pre-mRNA display unique biological activities and are subject to distinct regulatory controls. For example, the short and long isoforms of Bcl-x have opposing functions in apoptosis [30]. Genes whose protein isoforms change during tumorigenesis regulate major hallmarks of neoplasia, including: oncogenes such as Kras [31] and Wnt [32]; tumor suppressor genes such as Brca1 [33] and Mdm2 [34]; genes regulating apoptosis such as Bcl2, Fas, and caspase 2 [35]; genes regulating angiogenesis such as $V E G F[36]$; genes involved in DNA repair such as DNA polymerase $\beta$ [37]; genes regulating cell adhesion and intercellular communication such as CD44 [16] and cadherin-11 [38]; and genes involved in cellular senescence such as the INK4a locus $\left(p 14^{A R F}\right.$ and p16 ${ }^{I N K 4 a}$ ) [39].

The best-characterized example of how hnRNP A1 and ASF/SF2 may modulate mRNA alternative splicing is CD44, a membrane glycoprotein that binds hyaluronic acid and osteopontin and is involved in cell-cell and cell-extracellular matrix interactions $[16,18]$. CD44 has multiple alternative splice forms, with particular variants associated with metastasis. These variant species influence primary tumor growth and enable tumor cells circulating in the vasculature to adhere to the vascular endothelium, thereby facilitating tumor seeding at distant sites. In vitro studies show that hnRNP A1 binds regulatory splice elements in CD44 variant exon v5 and modulates its cancer-associated alternative splicing [40]. Changes in the concentrations of ASF/SF2 correspond to altered CD44 splicing in mouse mammary gland tumorigenesis [15] and human colon adenocarcinoma [27]. We describe herein a substantial increase in the metastatic-associated CD44 mRNA isoforms in lung tumors compared to regenerative proliferating lung, uninvolved lung, and control tissue. The parallel time course of increased hnRNP A1 and ASF/SF2 expression with appearance of the variant CD44 isoforms suggests that hnRNP A1 and ASF/SF2 may be involved in these splicing changes. There are numerous additional splicing factors, however, and changes in one or several of these may also impact expression of variant CD44 isoforms. We are currently conducting in vitro studies to delineate any causal role between aberrant hnRNP A1 and ASF/SF2 expression and alternative splicing of CD44 as well as other cancer-associated genes. 
In summary, numerous enhancing and repressing proteins interact in a combinatorial fashion with premRNA and the spliceosome to direct splice-site selection $[3,4]$. Because of this complexity, very little is known about this process in a whole animal model or how it becomes dysregulated during disease states including cancer. The intriguing changes in hnRNP A1 and ASF/SF2 in mouse lung tumors described herein are the beginnings of an effort to characterize the role of pre-mRNA alternative splicing in lung neoplasia. This unique neoplastic expression profile of splicing factors could serve as a diagnostic tool for identifying early lung cancer as well as distinguishing lung cancer subtypes.

\section{ACKNOWLEDGMENTS}

We thank Ms. Katherine A. Peebles for her helpful comments on this manuscript. This study was supported by USPHS CA33497 and CA 96133 (to A.M.M).; FIS/00/0835, PI021116, and RTIC C03/10 (to L.M.M. and R.P.), and University of Colorado Cancer Center Seed Grant (to L.K.Z).

\section{REFERENCES}

1. Stewart BW, Kleihues P. World Cancer Report. Lyon: IARCPress; 2003.

2. Mercatante $D$, Kole R. Modification of alternative splicing pathways as a potential approach to chemotherapy. Pharmacol Ther 2000;85:237-243.

3. Dreyfuss G, Kim VN, Kataoka N. Messenger-RNA-binding proteins and the messages they carry. Nat Rev Mol Cell Biol 2002;3:195-205.

4. Black DL. Mechanisms of alternative pre-messenger RNA splicing. Annu Rev Biochem 2003;72:291-336.

5. Graveley BR. Alternative splicing: Increasing diversity in the proteomic world. Trends Genet 2001;17:100-107.

6. Modrek B, Resch A, Grasso C, Lee C. Genome-wide detection of alternative splicing in expressed sequences of human genes. Nucleic Acids Res 2001;29:2850-2859.

7. Wang ZN, Lo HS, Yang $\mathrm{H}$, et al. Computational analysis and experimental validation of tumor-associated alternative RNA splicing in human cancer. Cancer Res 2003;63:655657.

8. Tockman MS, Mulshine $J \mathrm{~L}$, Piantadosi $\mathrm{S}$, et al. Prospective detection of preclinical lung cancer: Results from two studies of heterogeneous nuclear ribonucleoprotein A2/B1 overexpression. Clin Cancer Res 1997:3:2237-2246.

9. Pino I, Pio R, Toledo $G$, et al. Altered patterns of expression of members of the heterogeneous nuclear ribonucleoprotein (hnRNP) family in lung cancer. Lung Cancer 2003;41:131143.

10. Peebles KA, Duncan MW, Ruch RJ, Malkinson AM. Proteomic analysis of a neoplastic mouse lung epithelial cell line whose tumorigenicity has been abrogated by transfection with the gap junction structural gene for connexin 43, Gjal. Carcinogenesis 2003:24:651-657.

11. Blaxall BC, Dwyer-Nield L, Bauer AK, Bohlmeyer TJ, Malkinson AM, Port JD. Differential expression and localization of the mRNA binding proteins, AU-rich element mRNA binding protein (AUF-1) and Hu antigen $\mathrm{R}(\mathrm{HuR})$, in neoplastic lung tissue. Mol Carcinog 2000;28:76-83.

12. Malkinson AM. Primary lung tumors in mice as an aid for understanding, preventing, and treating human adenocarcinoma of the lung. Lung Cancer 2001;32:265-279.
13. Mayeda A, Krainer AR. Regulation of alternative pre-mRNA splicing by hnRNP A1 and splicing factor SF2. Cell 1992;68: 365-375.

14. Caceres JF, Stamm S, Helfman DM, Krainer AR. Regulation of alternative splicing in vivo by overexpression of antagonistic splicing factors. Science 1994;265:1706-1709.

15. Stickeler E, Kittrell F, Medina D, Berget SM. Stagespecific changes in SR splicing factors and alternative splicing in mammary tumorigenesis. Oncogene 1999;18:35743582.

16. Cooper DL, Dougherty GJ. To metastasize or not? Selection of CD44 splice sites. Nat Med 1995;1:635-637.

17. Hirata T, Fukuse T, Naiki H, Hitomi S, Wada H. Expression of CD44 variant exon 6 in stage I non-small cell lung carcinoma as a prognostic factor. Cancer Res 1998;58:1108-1110.

18. Ponta H, Sherman L, Herrlich PA. CD44: From adhesion molecules to signalling regulators. Nat Rev Mol Cell Biol 2003;4:33-45.

19. Malkinson AM, Koski KM, Evans WA, Festing MF. Butylated hydroxytoluene exposure is necessary to induce lung tumors in BALB mice treated with 3-methylcholanthrene. Cancer Res 1997;57:2832-2834.

20. Malkinson AM. Prevention of butylated hydroxytolueneinduced lung damage in mice by cedar terpene administration. Toxicol Appl Pharmacol 1979;49:551-560.

21. Lowry $\mathrm{OH}$, Rosebrough NJ, Farr AL, Randall RJ. Protein measurement with the folin phenol reagent. J Biol Chem 1951;193:265-275.

22. Malkinson AM, Beer DS. Major effect on susceptibility to urethan-induced pulmonary adenoma by a single gene in BALB/cBy mice. J Natl Cancer Inst 1983;70:931-936.

23. Witschi H, Malkinson AM, Thompson JA. Metabolism and pulmonary toxicity of butylated hydroxytoluene (BHT). Pharmacol Ther 1989;42:89-113.

24. Malkinson AM, Thaete LG, Blumenthal EJ, Thompson JA Evidence for a role of tert-butyl hydroxylation in the induction of pneumotoxicity in mice by butylated Hydroxytoluene. Toxicol Appl Pharmacol 1989;101:196-204.

25. Adamson IY, Bowden DH, Cote MG, Witschi H. Lung injury induced by butylated hydroxytoluene: Cytodynamic and biochemical studies in mice. Lab Invest 1977;36:26-32.

26. Thaete LG, Gunning WT, Stoner GD, Malkinson AM. Cellular derivation of lung-tumors in sensitive and resistant strains of mice-Results at 28 and 56 weeks after urethane treatment. J Nat Cancer Inst 1987;78:743-749.

27. Ghigna C, Moroni M, Porta C, Riva S, Biamonti G. Altered expression of heterogenous nuclear ribonucleoproteins and $\mathrm{SR}$ factors in human colon adenocarcinomas. Cancer Res 1998;58:5818-5824.

28. Xiao SH, Manley JL. Phosphorylation of the ASF/SF2 RS domain affects both protein-protein and protein-RNA interactions and is necessary for splicing. Genes Dev 1997;11: 334-344.

29. Caceres JF, Screaton GR, Krainer AR. A specific subset of SR proteins shuttles continuously between the nucleus and the cytoplasm. Genes Dev 1998;12:55-66

30. Boise LH, Gonzalezgarcia M, Postema CE, et al. BCl-X, A BCl2-related gene that functions as a dominant regulator of apoptotic cell-death. Cell 1993;74:597-608.

31. Wang $Y$, You M, Wang $Y$. Alternative splicing of the K-ras gene in mouse tissues and cell lines. Exp Lung Res 2001;27: 255-267.

32. Katoh M, Kirikoshi H, Saitoh T, Sagara N, Koike J. Alternative splicing of the WNT-2B/WNT-13 gene. Biochem Biophys Res Commun 2000;275:209-216.

33. Orban $\mathrm{TI}$, Olah E. Expression profiles of BRCA1 splice variants in asynchronous and in G1/S synchronized tumor cell lines. Biochem Biophys Res Commun 2001;280:32-38.

34. Lukas J, Gao DQ, Keshmeshian M, et al. Alternative and aberrant messenger RNA splicing of the $\mathrm{mdm} 2$ oncogene in invasive breast cancer. Cancer Res 2001;61:3212-3219. 
35. Jiang ZH, Wu JY. Alternative splicing and programmed cell death. Proc Soc Exp Biol Med 1999:220:64-72.

36. Yuan A, Yu CJ, Kuo SH, et al. Vascular endothelial growth factor 189 mRNA isoform expression specifically correlates with tumor angiogenesis, patient survival, and postoperative relapse in non-small-cell lung cancer. J Clin Oncol 2001;19: 432-441.

37. Thompson TE, Rogan PK, Risinger JI, Taylor JA. Splice variants but not mutations of DNA polymerase beta are common in bladder cancer. Cancer Res 2002;62:32513256 .
38. Feltes CM, Kudo A, Blaschuk O, Byers SW. An alternatively spliced cadherin-11 enhances human breast cancer cell invasion. Cancer Res 2002;62:6688-6697.

39. Zhu DG, Xu G, Ghandhi S, Hubbard K. Modulation of the expression of $p 16$ (INK4a) and $p 14(A R F)$ by hnRNP A1 and A2 RNA binding proteins: Implications for cellular senescence. J Cell Physiol 2002;193:19-25.

40. Matter N, Marx M, Weg-Remers S, Ponta H, Herrlich P, Konig $H$. Heterogeneous ribonucleoprotein $A 1$ is part of an exonspecific splice-silencing complex controlled by oncogenic signaling pathways. J Biol Chem 2000;275:35353-35360. 